\title{
TOEPLITZ AND HANKEL OPERATORS ON BARGMANN SPACES
}

by JAN JANAS

(Received 19 May, 1987)

1. Introduction. Let $\mu$ be the Gaussian measure in $\mathbb{C}^{n}$ given by $d \mu(z)=$ $(2 \pi)^{-n} \exp \left(-|z|^{2} / 2\right) d V$, where $d V$ is the ordinary Lebesgue measure in $\mathbb{C}^{n}$. The Segal-Bargmann space $H^{2}(\mu)$ is the space of all entire functions on $\mathbb{C}^{n}$ that belong to $L^{2}(\mu)$-the usual space of Gaussian square-integrable functions. Let $P$ be the orthogonal projection from $L^{2}(\mu)$ onto $H^{2}(\mu)$. For a measurable function $\varphi$ on $\mathbb{C}^{n}$, the multiplication operator $M_{\varphi}$ on $L^{2}(\mu)$ is defined by $M_{\varphi} h=\varphi h$. The Toeplitz operator $T_{\varphi}$ is defined on $H^{2}(\mu)$ by

$$
T_{\varphi} f=P M_{\varphi} f, \quad f \in H^{2}(\mu) .
$$

Some results on Toeplitz operators have been found by Berezin, Howe and others, [1], [6]. However more systematic study of these operators has been given by Berger and Coburn in their recent joint works [2], [3]. We thank them for sending us these works. In particular the description of the $C^{*}$-algebra generated by the Weyl form of the canonical commutation relations as uniform limits of almost periodic Toeplitz operators is given in [2]. Moreover [3] completely characterized the largest ${ }^{*}$-algebra $Q$ in $L^{\infty}\left(\mathbb{C}^{n}\right)$ for which $T_{f} T_{g}-T_{f g}$ is a compact operator for all $f, g$ in $Q$.

In this paper we concentrate on the following questions: under what conditions on the symbol $\varphi$ is the corresponding Toeplitz operator $P M_{\varphi} P$ a bounded, compact or in the Schatten-von Neumann class $\mathscr{C}_{p}, p \geq 1$. We give such conditions in terms of the Berezin transform $\tilde{\varphi}$ of $\varphi$. The question of boundedness and compactness of $T_{\varphi}$ has also been considered in [3]. Since $T_{f g}-T_{f} T_{g}=P M_{f}(I-P) M_{g} P$, there also naturally appears in this context the Hankel operator $H_{f}=(I-P) M_{f} P$. Therefore analogous questions will be answered for Hankel operators. Note that our results concerning Hankel operators are not covered by the recent theory of Hankel forms given by S. Janson, J. Peetre, R. Rochberg in [7]. We also find the spectrum of a class of bounded Toeplitz operators and consider the behaviour of Hankel operators under the action of the Weyl representation of $\mathbb{C}^{n}$.

The notation given below will be used throughout the paper. For any $p \geq 1, L^{p}\left(\mathbb{C}^{n}\right)$ stands for the usual $L^{p}$ space with respect to Lebesgue measure $d V$ in $\mathbb{C}^{n}$. We shall also use the obvious generalization $L^{p}(\rho d V)$, where $\rho$ is a function on $\mathbb{C}^{n}$. The scalar product of $\lambda, z \in \mathbb{C}^{n}$ is denoted by $(\lambda, z)$, but the same notation $(f, g)$ will be used for $f, g \in L^{2}(\mu)$. For $\lambda, z \in \mathbb{C}^{n}, e_{\lambda}(z)=e^{(z, \lambda) / 2}$ and let $k_{\lambda}(z)=e^{(z, \lambda) / 2-\left(|\lambda|^{2 / 4}\right)}$ be the normalized reproducing kernel for $H^{2}(\mu)$. The Berezin transform of a function $\varphi$ on $\mathbb{C}^{n}$ such that $\varphi k_{\lambda} \in L^{2}(\mu)$ for every $\lambda \in \mathbb{C}^{n}$ is given by

$$
\bar{\varphi}(\lambda)=\left(\varphi k_{\lambda}, k_{\lambda}\right) .
$$

For a function $f$ on $\mathbb{C}^{n}, q \in \mathbb{R}, a \in C^{n}$ we denote by $f(q \cdot)$ and $f(\cdot-a)$ the functions

Glasgow Math. J. 30 (1988) 315-323. 
$z \rightarrow f(q z)$ and $z \rightarrow f(z-a)$, respectively. The class of compact operators is always denoted by $\mathscr{K}$ and the Schatten-V. Neumann operators by $\mathscr{C}_{p} . C_{0}$ stands for the space of continuous functions on $\mathbb{C}^{n}$ which vanish at infinity.

For an $n$-tuple $k=\left(k_{1}, \ldots, k_{n}\right)$ of non-negative integers the standard basis vector $e_{k}$ in $H^{2}(\mu)$ is given by

$$
\begin{aligned}
& e_{k}(z)=\left(2^{|k|} k !\right)^{-1 / 2} z^{k}, \quad \text { where } \\
& k !=k_{1} ! k_{2} ! \ldots ! k_{n} !, \quad k=k_{1}+\ldots+k_{n} \text { and } \\
& z^{k}=z_{1}^{k_{1}} \cdots z_{n}^{k_{n}} .
\end{aligned}
$$

All other notations will be those commonly used in operator theory.

2. Toeplitz operators. We start with a simple proof of a sufficient condition for the boundedness of $T_{\varphi}$. A similar result is also contained in [3] but with a different proof.

Lemma 1. Suppose that $\varphi$ is measurable and $\bar{\varphi}(\sqrt{2} \cdot) \in L^{\infty}\left(\mathbb{C}^{n}\right)$. Then $P M_{\varphi} P$ is bounded and $\left\|P M_{\varphi} P\right\| \leq(8 \pi)^{n}\|\tilde{\varphi}(\sqrt{2} \cdot)\|_{\infty}$. Conversely, if $T_{\varphi}$ is bounded, then $\tilde{\varphi}(\cdot) \epsilon$ $L^{\infty}\left(\mathbb{C}^{n}\right)$

Proof. Note that $P M_{\varphi} P$ is the integral operator with the kernel $R(z, u)$ given by

$$
R(z, u)=\int \varphi(\xi) \overline{e_{\xi}(u) e_{z}(\xi)} d \mu(\xi)
$$

Let $C_{\varphi}$ be the integral operator with the kernel

$$
K(z, \mu)=\int|\varphi(\xi)| e^{\operatorname{Re}[\xi,(u+z) / 2]} d \mu(\xi) .
$$

Since $\left|P M_{\varphi} P f(z)\right| \leq C_{\varphi}|f|(z)$ it is clear that boundedness of $C_{\varphi}$ in $L^{2}(\mu)$ implies the boundedness of $P M_{\varphi} P$ in $L^{2}(\mu)$. Applying the Schur test for $C_{\varphi}$ we put $p(z)=q(z)=$ $e^{|z|^{2 / 2}}$, see [5, Theorem 5.2]. We have

$$
\begin{aligned}
& \int K(z, u) p(u) d \mu(u)=\iint|\varphi(\xi)| e^{-\left(|\xi-u|^{2} / 4\right)+\left(|\xi|^{2 / 4}\right)+\operatorname{Re}(\xi, z) / 2} d V(u) d \mu(\xi) \\
& =c_{n} \int|\varphi(\xi)| e^{-\left(|\xi|^{2 / 4)+\operatorname{Re}(\xi, z) / 2} d V(\xi)\right.} \\
& =c_{n} p(z)|\tilde{\varphi}(\sqrt{2} \cdot)|\left(\frac{z}{\sqrt{2}}\right),
\end{aligned}
$$

where

$$
c_{n}=2^{n} \int e^{-|w|^{2 / 4}} d V(w)=(8 \pi)^{n}
$$


Hence $P M_{\varphi} P$ is bounded and $\left\|P M_{\varphi} P\right\| \leq c_{n}\||\varphi(\sqrt{2} \cdot)|\|_{\infty}$. Since the converse is obvious by the definition of $\tilde{\varphi}$ the proof is complete.

Remark 1. Note that $|\tilde{\varphi}|(\sqrt{2} \cdot) \in L^{\infty}\left(\mathbb{C}^{n}\right)$ implies $|\tilde{\varphi}| \in L^{\infty}\left(\mathbb{C}^{n}\right)$. There are no bounded Toeplitz operators $T_{\varphi}$ with entire symbol $\varphi$ (by Liouville's Theorem). However, there are many bounded Toeplitz operators with unbounded symbol. We shall see below that unbounded symbols can even define trace class Toeplitz operators.

Our next result gives a sufficient condition for compactness of $T_{\varphi}$, also expressed in terms of Berezin symbol. Namely following the proof of Theorem $\mathrm{C}$ in [3] we shall prove

Proposition 2. If $|\tilde{\varphi}(\sqrt{2} \cdot)| \in C_{0}$, then $T_{\varphi} \in \mathscr{K}$.

Proof. First note $T_{|\varphi|} \in \mathscr{K}$ implies that $T_{\varphi} \in \mathscr{K}$. We claim that $T_{|\varphi|} \in \mathscr{K}$. Let $\chi_{r}$ be the characteristic function of $\{z,|z|>r\}$. Then

$$
T_{|\varphi|}=T_{|\varphi|\left(1-x_{r}\right)}+T_{|\varphi| x} .
$$

Since $T_{|\varphi|\left(1-\chi_{r}\right)}$ is compact we only have to show that

$$
\lim _{r \rightarrow \infty}\left\|T_{|\varphi| x,}\right\|=0 .
$$

By Lemma 1 it is enough to check that

$$
\lim _{r \rightarrow \infty}\left\|g_{r}\right\|_{\infty}=0
$$

where $g_{r}(\lambda)=\sqrt{|\varphi| \chi_{r}}(\sqrt{2} \cdot)(\lambda)$. But $g_{r} \in C_{0}, g_{r}(z) \leq g_{s}(z)$, when $r>s$ and $\lim _{r \rightarrow \infty} g_{r}(z)=0$ for every $z \in \mathbb{C}^{n}$. Hence by Dini's Theorem (+) holds and this completes the proof.

REMARK 2. This proof is exactly the same as the proof of the implication $|\varphi|^{2} \in C_{0} \Rightarrow M_{\varphi} P \in \mathscr{K}$ given in [3]. It turns out that using the integral expression of $T_{\varphi}$ one can also give sufficient conditions (in terms of Berezin symbol of $\varphi$ ) for the inclusions $T_{\varphi} \in \mathscr{C}_{p}, p \geq 2$ and $T_{\varphi} \in \mathscr{C}_{1}$.

Proposition 3. Let $p \geq 2$ and $p^{-1}+q^{-1}=1$. If $|\tilde{\varphi}|^{q}\left(\frac{\cdot}{\sqrt{4-q}}\right)\left(\frac{2}{\sqrt{4-q}} \cdot\right) \epsilon$ $L^{P-1}\left(\mathbb{C}^{n}, \rho d V\right)$, where $\rho(z)=\exp \left[\left(q^{2}-4\right)(4-q)^{-1}(q-1)^{-1}|z|^{2} 2^{-1}\right]$ then $T_{\varphi} \in \mathscr{C}_{p}$.

Proof. Let $S_{\varphi}$ be the integral operator with the kernel $R(z, u)$ given in the proof of Lemma 1. We have $S_{\varphi \mid H^{2}(\mu)}=T_{\varphi}$. Applying the Theorem of Russo [9], it is enough to check that

$$
\int\left[\int|R(z, u)|^{q} d \mu(u)\right]^{p-1} d \mu(z)<+\infty
$$


But this integral is less then

$$
\begin{aligned}
\int\left[\int\left((2 \pi)^{-n} \int|\varphi(\xi)| e^{-\mid \xi-(z+u)^{2} / 4} d V(\xi)\right)^{q} e^{q|(u+z) / 2|^{2}} d \mu(u)\right]^{p-1} d \mu(z) \\
=\int\left[\int|\tilde{\varphi}|^{q}\left(\frac{z+u}{2}\right) e^{q|(u+z) / 2|^{2}} d \mu(u)\right]^{p-1} d \mu(z)
\end{aligned}
$$

By direct computation we find that the last integral is equal

$$
c_{n} \int\left[\int|\tilde{\varphi}|^{q}\left(\frac{s}{\sqrt{4-q}}\right) e^{-1 / 2|s-(\sqrt{ } 4 / 4-q) z|^{2}} d V(s)\right]^{p-1} \rho(z) d V(z),
$$

where $\quad c_{n}=(4-q)^{n(1-p)}, \quad \rho(z)=\exp \frac{1}{2}\left[\left(q^{2}-4\right)(4-q)^{-1}(q-1)^{1}|z|^{2}\right]$. The proof is complete.

REMARK 3. Note that for $p=2$, the condition imposed on $\varphi$ reduces to a simple requirement: $|\tilde{\varphi}| \in L^{2}\left(\mathbb{C}^{n}\right)$.

Before we proceed further let us recall the following fact (contained in [1, p. 1137]). If $T_{\varphi} \in \mathscr{C}_{1}$ then $|\tilde{\varphi}| \in L^{1}\left(\mathbb{C}^{n}\right)$ and

$$
\operatorname{Tr} T_{\varphi}=\int \varphi d V
$$

Applying the above equality we have the following

Proposition 4. $T_{\varphi} \in \mathscr{C}_{1}$ if and only if $\varphi \in L^{1}\left(\mathbb{C}^{n}\right)$. If $T_{\varphi} \in \mathscr{C}_{1}$, then $\operatorname{Tr} T_{\varphi}=\int \varphi d V$.

Proof. By the above mentioned result of Berezin the "only if" part and the equality $\operatorname{Tr} T_{\varphi}=\int \varphi d V$ are obvious (note that $\int|\tilde{\varphi}| d V=\int|\varphi| d V$ ).

On the other hand suppose that $|\tilde{\varphi}| \in L^{1}\left(\mathbb{C}^{n}\right)$. Let $\left\{g_{K}\right\}$ be an arbitrary basis in $H^{2}(\mu)$. It is enough to check that

$$
\sum_{K}\left|\left(T_{\varphi} g_{K}, g_{K}\right)\right|<+\infty, \quad \text { see }[4] .
$$

We have

$$
\begin{aligned}
\sum_{K}\left|\left(T_{\varphi} g_{K}, g_{K}\right)\right| \leq \sum_{K}|\varphi|\left|\dot{g}_{K}\right|^{2} d \mu & =\sum_{K}\left(|\varphi| g_{K}, g_{K}\right)=\int\left(|\varphi| e_{z}, e_{z}\right) d \mu(z) \\
& =\int|\tilde{\varphi}| d V=\int|\varphi| d V
\end{aligned}
$$

This completes the proof.

REMARK 4 . In the case $1<p<2$ one can also find a certain sufficient condition for $T_{\varphi} \in \mathscr{C}_{p}$. However it is not formulated in terms of $\tilde{\varphi}$. Namely, if for the standard basis $e_{K}$ 
in $H^{2}(\mu)$,

$$
\dot{\int}|\varphi(z)|^{p} \sum_{K}\left|e_{k}(z)\right|^{p} d_{k}^{p-1} d \mu(z)<\infty, \quad \text { then } T_{\varphi} \in \mathscr{C}_{p}
$$

Here

$$
d_{k}=\int\left|e_{k}\right|^{q} d \mu=\frac{\Gamma\left(\frac{k q}{2}+1\right)}{(k !)^{q / 2}}, \quad \frac{1}{p}+\frac{1}{q}=1 .
$$

We conclude this part of the paper with a result concerning the spectrum $\sigma\left(T_{\varphi}\right)$ of $T_{\varphi}$, for a class of symbols. First some remarks on homogeneous polynomials. Let $P_{N}$ denote the space of homogeneous polynomials of degree $N$ in $\mathbb{C}^{n}$. It is clear that

$$
H^{2}(\mu)=\bigoplus_{N=0}^{\infty} P_{N}
$$

By a simple computation one can check that any $P_{N}$, considered as a finite dimensional subspace of $H^{2}(\mu)$, has the reproducing kernel $e_{z}^{(N)}$ given by

$$
e_{z}^{(N)}(a)=\frac{(a, z)^{N}}{2^{N} N !} .
$$

Now we are ready to formulate the next result.

PROPosition 5. If $T_{|\varphi|}$ is bounded and $\varphi\left(e^{i \theta} z\right)=\varphi(z)$ for every $\theta \in \mathbb{R}$, then

i) $T_{\varphi}=\bigoplus_{N=0}^{\infty}\left(T_{\varphi \mid P_{N}}\right)$
ii) $\sigma\left(T_{\varphi}\right)=\bigcup_{N=0}^{\infty} \sigma\left(T_{\varphi \mid P_{N}}\right)$

Proof.

i) Let $e_{K}$ be the standard basis in $H^{2}(\mu)$. Direct computation shows that

$$
\left(T_{\varphi} e_{K}, e_{1}\right)=e^{i(|k|-|1|) \theta}\left(T_{\varphi} e_{k}, e_{1}\right) .
$$

Hence $T_{\varphi} P_{N} \subseteq P_{N}$, for $N=0,1,2, \ldots$ Since $\bar{\varphi}\left(e^{i \theta} z\right)=\bar{\varphi}(z)$ and $T_{\varphi}^{*}=T_{\bar{\varphi}}$ this completes the proof of i).

ii) Denote by $T_{N}=T_{\varphi \mid P_{N}}$. We have to show that

$$
\sigma(T) \subseteq \overline{\bigcup_{N=0}^{\infty} \sigma\left(T_{N}\right)}
$$

because the opposite inclusion always holds.

Take $\lambda \notin \bigcup_{N=0}^{\infty} \sigma\left(T_{N}\right)$. There exists $\delta>0$ such that $r_{N}=\operatorname{dist}\left(\lambda, \sigma\left(T_{N}\right)\right) \geq \delta$ for every $N=0,1,2, \ldots$ Let \|\|$_{0}$ be any cross norm in the set of finite rank operators in $H^{2}(\mu)$. Suppose that \|\|$_{0}$ is not equivalent to the operator norm. Then by Lemma 3 of [8] we 
have

$$
\left\|\left(\lambda-T_{N}\right)^{-1}\right\| \leq \frac{3}{2} r_{N}^{-1} \exp \left[39 r_{N}^{-1}\left\|T_{N}\right\|_{0} \tau\left(r_{N} / 6\left\|T_{N}\right\|_{0}\right)\right],
$$

here $\tau: \mathbb{R}_{+} \rightarrow \mathbb{R}_{+}$is a decreasing function such that $\tau(r)=0$, for $r>1$.

We choose $\|A\|_{0}=\left(\operatorname{tr} A^{*} A\right)^{1 / 2}$. The above estimation $(*)$ shows that $\left\|\left(\lambda-T_{N}\right)^{-1}\right\|$ are uniformly bounded, if $\left\|T_{N}\right\|_{0}$ are uniformly bounded.

But

$$
\left\|T_{N}\right\|_{0}^{2}=\sum_{s, p=0}^{d(N)}\left|\int \varphi e_{p} \bar{e}_{s} d \mu\right|^{2},
$$

where $d(N)$ is finite and is equal to $\operatorname{dim} P_{N}$. By the Schwarz inequality it is enough to estimate

$$
\sum_{s=0}^{d(N)} \int|\varphi|\left|e_{s}\right|^{2} d \mu=\int|\varphi(z)| e_{z}^{(N)}(z) d \mu(z)
$$

Since $T_{|\varphi|}$ is bounded and $\int\left\|e_{\xi}^{(N)}\right\|^{2} d \mu(\xi)=c_{n}$, we have

$$
\int|\varphi(z)|\left(e_{z}^{(N)}, e_{z}^{(N)}\right) d \mu(z) \leq\left\|T_{|\varphi|}\right\| \int\left\|e_{\xi}^{(N)}\right\|^{2} d \mu(\xi)=c_{n}\left\|T_{|\varphi|}\right\| .
$$

Hence $\left\{\left\|T_{N}\right\|_{0}\right\}$ are uniformly bounded and this completes the proof. open.

The question how to describe the spectrum of $T_{\varphi}$ for more general $\varphi$ seems to be

3. Hankel operators. This part of the paper contains a few results concerning Hankel operators in $H^{2}(\mu)$. For a given measurable function $\varphi$ we define the Hankel operator by

$$
H_{\varphi} f=(I-P) M_{\varphi} f, \quad f \in H^{2}(\mu) .
$$

In the classical case of the unit disc there are plenty of bounded Hankel operators with anti-holomorphic symbol $\varphi$. However in the Bargmann space there are only trivial bounded Hankel operators with anti-holomorphic symbol. Indeed, if $H_{\varphi}$ is bounded, then for any $f \in H^{2}(\mu)$ we have $\bar{\varphi} f \in H^{2}(\mu)$, so by Remark $1 \varphi$ must be a constant. Nevertheless even for unbounded symbol $\varphi$ one can sometimes extend $H_{\varphi}$ given by $(\mathrm{H})$ on a dense domain $D$, to a bounded operator in $H^{2}(\mu)$. Namely applying the Schur test once more we have

Proposmion 6. If $H_{\varphi}$ is defined by $(H)$ on a dense domain $D$ and $\varphi$ satisfies the inequality

$$
|\varphi(z)-\varphi(w)| \leq M e^{|z-w|^{2 / 8}},
$$

then there exists a unique extension $\bar{H}_{\varphi}$ of $H_{\varphi}$ which is bounded in $H^{2}(\mu)$. Moreover 
$\left\|\bar{H}_{\varphi}\right\| \leq M c_{n}$, where

$$
c_{n}=(2 \pi)^{-n} \int e^{-|z|^{2 / 8}} d V
$$

Proof. Direct calculation shows that $H_{\varphi}$ is given on $D$ by the formula

$$
H_{\varphi} u(z)=\int[\varphi(z)-\varphi(w)] \overline{e_{z}(w)} u(w) d \mu(w)
$$

$u \in D$.

Define the integral operator $S_{\varphi}$ on $L^{2}(\mu)$ by

$$
S_{\varphi} f(z)=\int\left|[\varphi(z)-\varphi(w)] e_{z}(w)\right| f(w) d \mu(w)
$$

We claim that $S_{\varphi}$ is bounded in $L^{2}(\mu)$. Indeed, applying the Schur test for $p(z)=q(z)=$ $e^{|z|^{2 / 4}}$ and the kernel $S(z, w)=\left|[\varphi(z)-\varphi(w)] e_{z}(w)\right|$ we have by $(++)$

$$
\begin{array}{rl}
\int S(z, w) p(w) d \mu(w) \leq M \int e^{|z-w|^{2} / 8+\operatorname{Re}(z, w)+|w|^{2 / 4}} & d \mu(w) \\
& \leq(2 \pi)^{-n} M p(z) \int e^{-|z-w|^{2 / 8}} d V(w)=M c_{n} p(z)
\end{array}
$$

Hence $S_{\varphi}$ is bounded and $\left\|S_{\varphi}\right\| \leq M c_{n}$. Since

$$
\left|H_{\varphi} u(z)\right| \leq S_{\varphi}|u|(z)
$$

it follows that $H_{\varphi}$ has the unique bounded extension $\bar{H}_{\varphi}$ on $H^{2}(\mu)$ and $\left\|\bar{H}_{\varphi}\right\| \leq M c_{n}$. The proof is complete.

REMARK. The above proposition does not contradict our earlier comments on the lack of non-trivial bounded Hankel operators with anti-holomorphic symbols. This is clear because the extension $\bar{H}_{\varphi}$ of $H_{\varphi}$ from $D$ onto $H^{2}(\mu)$ is not given in general by $(H)$. For example, complex conjugate of polynomials satisfy $(++)$, but they don't define bounded $H_{\varphi}$ on $H^{2}(\mu)$ given by $(\mathrm{H})$.

Our next result concerns the question: when is $H_{\varphi} \in \mathscr{C}_{p}$ ? Applying once again the Theorem of Russo we shall find out when the above defined $S_{\varphi}$ is in $\mathscr{C}_{p}$. (and thus $\left.H_{\varphi} \in \mathscr{C}_{p}\right)$.

Proposition 7. Let $p \geq 2$ and $p^{-1}+q^{-1}=1$. If $\varphi \in L^{p}(\eta d V)$ and $|\bar{\varphi}|^{q}\left(\frac{q}{2} \cdot\right) \epsilon$ $L^{p-1}(\eta d V)$, where $\eta(z)=\exp \left[\left(p^{2}-4\right) 8^{-1}(p-1)^{-1}|z|^{2}\right]$, then $H_{\varphi} \in \mathscr{C}_{p}$. 
Proof. Applying the Theorem of Russo to $S_{\varphi}$, we have

$$
\begin{aligned}
& \left.\iiint|S(z, w)|^{q} d \mu(w)\right]^{p / q} d \mu(z) \\
& \quad \leq c_{q} \int\left[\int\left(|\varphi(z)|^{q}+|\varphi(w)|^{q}\right) e^{q \operatorname{Re}(w, z) / 2} d \mu(w)\right]^{p / q} d \mu(z) \\
& \leq c_{q}\left\{\left[\int\left(\int|\varphi(z)|^{q} e^{q \operatorname{Re}(w, z) / 2} d \mu(w)\right)^{p-1} d \mu(z)\right]^{1 /(p-1)}\right. \\
& \left.+\left[\int\left(\int|\varphi(w)|^{q} e^{q \operatorname{Re}(w, z) / 2} d \mu(w)\right)^{p-1} d \mu(z)\right]^{1 /(p-1)}\right\}^{p-1} .
\end{aligned}
$$

Direct calculation shows that under our assumptions on $\varphi$ both integrals in the brackets [ ] are finite.

This completes the proof.

Remark. For $p=2$ the assumptions of Proposition reduce simply to $\varphi \in L^{2}\left(\mathbb{C}^{n}\right)$ (because then $|\tilde{\varphi}|^{2} \in L^{1}\left(\mathbb{C}^{n}\right)$ automatically).

We conclude this work with a result concerning the behaviour of Hankel operators under the action of the Weyl representation of $\mathbb{C}^{n}$ in $L^{2}(\mu)$. Recall that the Weyl (unitary in $\left.L^{2}(\mu)\right)$ operators $W_{\lambda}, \lambda \in \mathbb{C}^{n}$ are defined by

$$
W_{\lambda} f(z)=k_{\lambda}(z) f(z-\lambda), \quad f \in L^{2}(\mu) .
$$

We have

Proposition 8. For any bounded Hankel operator $H_{\varphi}$ and $u \in H^{2}(\mu)$

$$
\left(W_{\lambda} H_{\varphi} W_{\lambda}^{-1} u\right)(z)=\left(H_{\varphi(\cdot-\lambda)} u\right)(z) .
$$

Proof. Applying the integral form of $H_{\varphi}$ and the equality

$$
\frac{d \mu(\cdot-w)}{d \mu}(a)=\left|k_{w}(a)\right|^{2},
$$

one can check $(* *)$ by direct calculation.

REMARK. The last result shows that any necessary and sufficient conditions concerning various properties of $H_{\varphi}$ (like $H_{\varphi} \in \mathscr{C}_{p}$ ) should be invariant under translations of the symbol $\varphi$.

\section{REFERENCES}

1. F. A. Berezin, Covariant and contravariant symbols of operators, Jzw. AN SSSR, 35 (1972), 1134-1167 (Russian).

2. C. A. Berger and L. A. Coburn, Toeplitz operators and quantum mechanics, J. Functional Analysis 68 (1986), 273-299. 
3. C. A. Berger and L. A. Coburn, Toeplitz operators on the Segal-Bargmann space, Trans. Amer. Math. Soc. 301 (1987), 813-829.

4. J. C. Gohberg and M. G. Krein, Introduction to the theory of non-selfadjoint operators in Hilbert space, (Nauka 1965), (Russian). 1978).

5. P. R. Halmos and V. S. Sunder, Bounded integral operators on $L^{2}$ spaces, (Springer-Verlag,

6. R. Howe, Quantum mechanics and partial differential equations J. Functional Analysis 38 (1980) $188-254$.

7. S. Janson, J. Peetre and R. Rochberg, Hankel forms and the Fock space (preprint-1986).

8. A. Pokrzywa, On continuity of spectra in norms ideals, Linear Algebra and Appl. 69 (1985) 121-130.

9. B. Russo, On the Hausdorff-Young theorem for integral operators, Pacific. J. Math. 68 (1977), 241-253.

\section{INSTYTUT MATEMATYCZNY}

PAN

31-027 KRAKOW

Ul. Solskiego 30

PolsKa. 